\title{
Blue Phosphorescent Organic Light-Emitting Devices with the Emissive Layer of mCP:FCNIr(pic)
}

\author{
Ji Geun Jang and Hyun Jin Ji \\ Department of Electronics Engineering, Dankook University, San 29, Anseo-dong, Cheonan, Chungnam 330-714, Republic of Korea \\ Correspondence should be addressed to Ji Geun Jang, semicgk@dankook.ac.kr
}

Received 15 February 2012; Accepted 17 April 2012

Academic Editor: Etienne Baranoff

Copyright (๑) 2012 J. G. Jang and H. J. Ji. This is an open access article distributed under the Creative Commons Attribution License, which permits unrestricted use, distribution, and reproduction in any medium, provided the original work is properly cited.

\begin{abstract}
New high-efficiency blue-light-emitting phosphorescent devices with $300 \AA$-thick emissive layer of N,N'-dicarbazolyl-3,5benzene $[\mathrm{mCP}]$ doped with $10 \mathrm{vol} \%$ bis[(3,5-difluoro-4-cyanophenyl)pyridine]iridium picolinate [FCNIr(pic)] were fabricated with the different treatments of hole and electron transport layers. In the experiments, a single layer of 1,1-bis-(di-4polyaminophenyl)cyclohexane [TAPC] and a double layer of $\mathrm{N}, \mathrm{N}^{\prime}-\operatorname{di}\left(1\right.$-naphthyl)-N, $\mathrm{N}^{\prime}$-diphenylbenzidine $[\mathrm{NPB}]$ and $\mathrm{mCP}$ were used as hole transport layers (HTLs). In addition, $500 \AA$-thick double layers of tris-[3-(3-pyridyl)mesityl]borane [3TPYMB] and 4,7-diphenyl-1,10-phenanthroline [Bphen] were used as electron transport layers (ETLs) with various thickness combination of 3 TPYMB/Bphen. Among the fabricated devices, the one using TAPC as an HTL and 3 TPYMB $(100 \AA) / B p h e n(400 \AA)$ as an ETL showed best electroluminescent characteristics with a maximum quantum efficiency of $13.3 \%$ and a luminance of $950 \mathrm{~cd} / \mathrm{m}^{2}$ at $10 \mathrm{~V}$. The color coordinates were $(0.14,0.22)$ on the Commission Internationale de I'Eclairage (CIE) chart, and the electroluminescent spectra showed the double-peak emissions at $458 \mathrm{~nm}$ and $483 \mathrm{~nm}$.
\end{abstract}

\section{Introduction}

Organic light-emitting devices (OLEDs) have been intensively investigated for their applications in the solid-state lightings as well as full-color displays $[1,2]$. Though the blue phosphorescent organic light-emitting diodes (PhOLEDs) are essential for the development of all phosphorescent active matrix OLEDs, highly efficient blue PhOLEDs are hard to obtain due to the large energy gap of dopants, which lead to insufficient carrier injection and exciton confinement. In the blue PhOLEDs, triplet of dopant should be larger than $2.7 \mathrm{eV}$ for blue emission. Therefore, the use of host with wide energy gap is necessary because triplet of host shoud be larger than that of dopant in the hostdopant system of an emissive layer. The well-known host material in the blue PhOLEDs is mCP. It has a good hole transport property due to a carbazole unit in the backbone structure and a large triplet of $2.9 \mathrm{eV}$ for efficient energy transfer [3]. Iridium(III) bis((4,6-difluorophenyl)pyridinate- $\left.\mathrm{N}, \mathrm{C}^{\prime}\right)$ picolinate [FIrpic] is the most well-known blue phosphorescent dopant [4]. Kawamura et al. demonstrated that the photoluminescence internal quantum yield of the blue emitter of FIrpic could approach nearly 100\% when doped into the wide energy gap host of mCP [5]. However, the theoretical electrophosphorescence is difficult to carry out because of the lack of highly efficient carrier transport materials with wide energy gap for sufficient carrier injection and exciton confinement. Furthermore, color performances of the FIrpic-based devices were poor with the vertical coordinates of more than 0.3 on the CIE chart.

The quantum efficiency remarkably decreases due to carrier injection loss, nonradiative relaxation of excitons, triplet-triplet annihilation at high current density, and so forth $[6,7]$. The triplets have rather long lifetime so that they may diffuse to the neighbor layers by passing through an emissive layer. This effect also results in the declination of luminous efficiency and color purity due to energy transfer and relaxation of excitons outside the emissive layer. Therefore, the structural design of PhOLEDs which can 


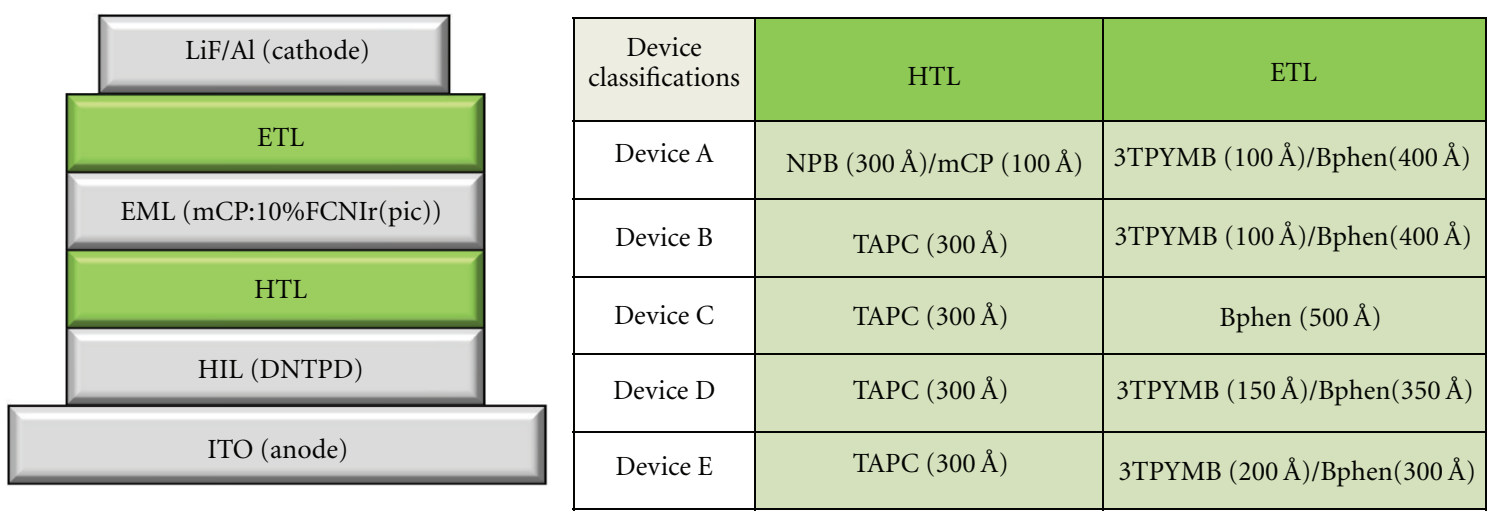

FIgURE 1: Basic structure and classification of the fabricated devices.

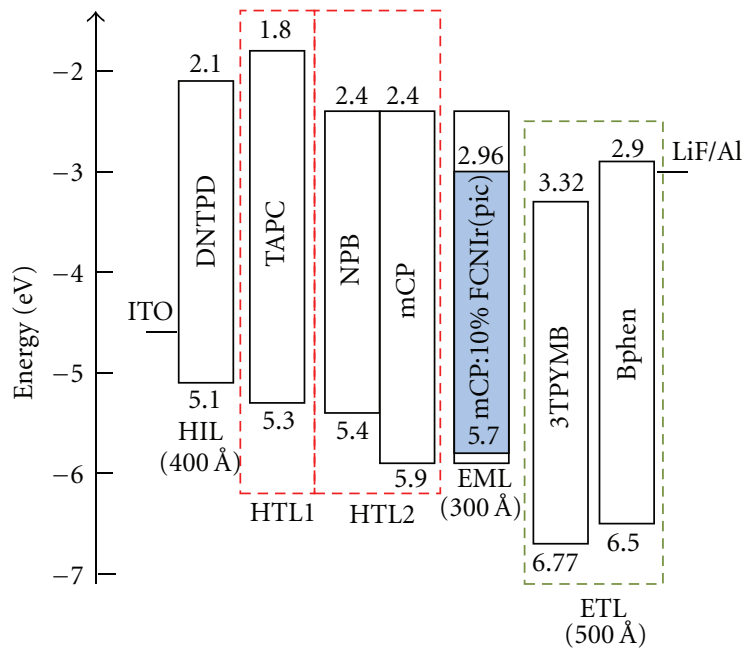

Figure 2: Energy band diagram of the used materials.

confine the triplet excitons in an emissive layer is extremely important to obtain high-efficiency devices.

Yook et al. reported a deep blue PhOLED with structure of $\mathrm{N}, \quad \mathrm{N}^{\prime}$-diphenyl-N,N'-bis-[4-(phenyl-m-tolylamino)phenyl]-biphenyl-4,4'-diamine[DNTPD]/NPB/mCP /mCP: tris[(3,5-difluoro-4-cyanophenyl)pyridine]iridium[FCNIr]/ Bphen in which a quantum efficiency of $9.2 \%$ and a current efficiency of $11 \mathrm{~cd} /$ A were obtained with a color coordinate of $(0.15 .0 .16)$ at $500 \mathrm{~cd} / \mathrm{m}^{2}$ [8]. The FCNIr has a wide triplet of $2.8 \mathrm{eV}$ for deep blue emission due to a strong electron withdrawing $\mathrm{CN}$ substituent besides $F$ units.

In this work, new high efficiency blue-light-emitting phosphorescent devices with an emissive layer of mCP doped with $\mathrm{FCNIr}$ (pic) were fabricatedand evaluated according to the treatments of hole and electron transport layers.

In the experiments, the electroluminescent characteristics of the devices with a single layer of TAPC and a double layer of $\mathrm{NPB} / \mathrm{mCP}$ as an HTL were compared. In addition, the double layers of $3 \mathrm{TPYMB} / \mathrm{Bphen}$ were used as an electron transport layers (ETLs) with various thickness combinations of 3TPYMB/Bphen. To our knowledge, the use of the mCP : FCNIr(pic) emitter as well as the adoption of the
TAPC as an HTL and the 3TPYMB/Bphen double layer as an ETL in the blue PhOLEDs was firstly attempted in this paper.

\section{Experimental Procedure}

The substrates with an ITO (indium tin oxide) anode of $12 \Omega /$ sq on glass were cleaned by ultrasonic cleaning process with acetone and isopropyl alcohol. The remaining solvent was removed by soft baking for 10 minutes at $100^{\circ} \mathrm{C}$. To improve the surface morphology of ITO transparent electrode film, the substrates were plasma treated at $150 \mathrm{~W}$ for two minutes under $8 \mathrm{~m}$ Torr pressure of $\mathrm{O}_{2} / \mathrm{Ar}$. The plasma treatment before deposition of the first organic layer is expected to reduce the energy barrier for hole injection from anode and remove the surface contaminants. All organic layers and cathode layers were deposited by insitu method under $5 \times 10^{-8}$ Torr.

As a sequence of deposition process, the DNTPD with thickness of $400 \AA$ was firstly deposited as a hole injection layer. Then, two kinds of HTLs were used according to device classifications: a conventional structure of $\operatorname{NPB}(300 \AA) / \mathrm{mCP}(100 \AA)$ was used in the device $\mathrm{A}$ and a single layer of TAPC $(300 \AA)$ in the other devices. Next, $300 \AA$ thick mCP doped with $10 \% \mathrm{FCNIr}$ (pic) as a volume ratio was deposited as an emissive layer. In the formation of ETLs, the 3TPYMB/Bphen with a total thickness of $500 \AA$ was deposited with various 3 TPYMB thicknesses according to the device classifications: the thicknesses of 3TPYMB were $100 \AA$ in the devices $(\mathrm{A}, \mathrm{B}), 0 \AA$ in the device $\mathrm{C}, 150 \AA$ in the device $\mathrm{D}$, and $200 \AA$ in the device E. Finally, $10 \AA$-thick LiF and $1200 \AA$-thick Al were successively deposited as a cathode. The structures of the fabricated devices and energy diagram of the used materials are shown in Figures 1 and 2.

\section{Results and Discussion}

In the energy diagram of Figure 2, the DNTPD is well known as a hole injection material with the HOMO level of $5.1 \mathrm{eV}$ and the LUMO level of $2.1 \mathrm{eV}$ [9]. The electrons from the anode of indium tin oxide (ITO) are easily injected into the DNTPD because its highest occupied molecular orbital (HOMO) level is similar to Fermi level of ITO. The 


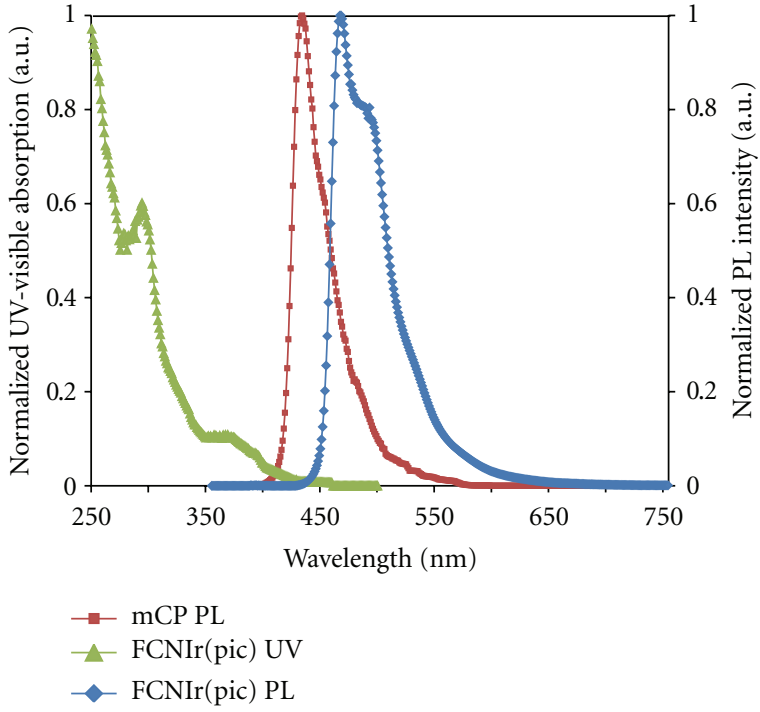

Figure 3: UV-visible absorption and PL spectra of FCNIr(pic) including the PL spectra of $\mathrm{mCP}$.

TAPC as a hole transport material has the HOMO level of $5.3 \mathrm{eV}$ and the LUMO level of $1.8 \mathrm{eV}$ [10]. The triplet energy of TAPC is $2.87 \mathrm{eV}$ [11] which is larger than that of FCNIr(pic) so that the triplet excitons can be effectively confined without diffusion loss into the HTL. Moreover, the electrons injected from cathode are confined in the EML due to the large LUMO offset $(\sim 0.6 \mathrm{eV})$ at the interface of the TAPC and the emissive layer. Therefore, the TAPC can be used as a good HTL in the high-efficiency blue PhOLED. With the same condition except HTL in the device structure, the electroluminescent characteristics of the device with the TAPC as an HTL were at firstly compared with those of the device with the conventional $\mathrm{NPB} / \mathrm{mCP}$ as an HTL. And then, the composition effect of 3TPYMB/Bphen as an ETL on the electroluminescent characteristics of the devices with the TAPC as an HTL was investigated. Both Bphen and 3TPYMB have been used as a material of ETL in the conventional OLEDs. The triplet energy of Bphen is $2.5 \mathrm{eV}$ [12] so that the Bphen cannot sufficiently confine the excitons. On the other hand, 3TPYMB has a triplet energy of $2.87 \mathrm{eV}$ which is higher than the exciton energy of FCNIr(pic) and a deep HOMO level of $6.77 \mathrm{eV}$ [13]. Therefore, the use of 3 TPYMB as an ETL can expect an effective confinement of the excitons as well as the injected holes in the emissive layer. However, the electron mobility of 3TPYMB is much lower than that of Bphen [14]. To solve these problems, the bilayer of 3TPYMB/Bphen can be used as an ETL. The addition of a properly thick 3TPYMB to Bphen in the ETL structure can improve the electroluminescent characteristics in the blue PhOLEDs because the excitons and the injected holes are effectively confined in the emissive layer without a serious increase of current resistance.

Figure 3 shows the UV-visible absorption and photoluminescence (PL) spectra of FCNIr(pic). The PL emission peak of FCNIr(pic) was observed at $460 \mathrm{~nm}$ with a vibrational peak at $485 \mathrm{~nm}$. The FCNIr(pic) shows a deep blue

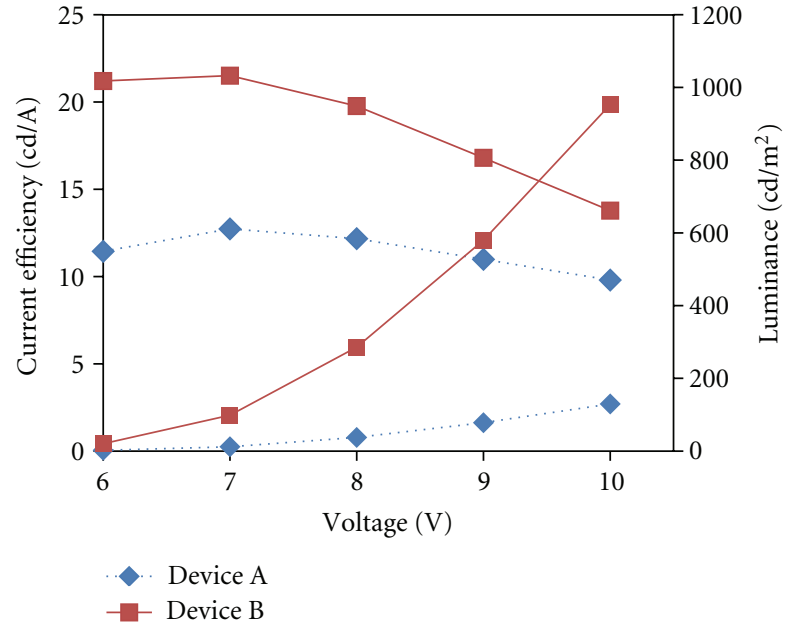

FIgURE 4: Current efficiency-voltage-luminance characteristics of the devices $(\mathrm{A}, \mathrm{B})$.

emission due to the strong electron withdrawing $\mathrm{CN}$ group in the phenyl unit of main ligand. The PL spectra of $\mathrm{mCP}$ are also shown in Figure 3. It can be clearly seen that PL spectra of $\mathrm{mCP}$ are overlapped with absorption of $\mathrm{FCNIr}$ (pic) in a range of $400 \sim 450 \mathrm{~nm}$. Therefore, the efficient energy transfer from $\mathrm{mCP}$ to $\mathrm{FCNIr}$ (pic) can be expected. In addition, Dexter energy transfer between the triplets of $\mathrm{mCP}$ and FCNIr(pic) can be occurred because the triplet of $\mathrm{mCP}$ is $2.9 \mathrm{eV}$ which is high compared to $2.72 \mathrm{eV}$ of FCNIr(pic) [15].

The electrical properties of the devices were measured using a Polaronix M6100 test system (McScience). The optical properties such as luminance, emission spectrum, and CIE color coordinates were evaluated using a CS-1000 spectro-radiometer (Konica Minolta) in a dark room.

The current efficiency-voltage-luminance characteristics of the devices (A, B) are shown in Figure 4. In Figure 4, the maximum current efficiencies were $12.7 \mathrm{~cd} / \mathrm{A}$ for the device $\mathrm{A}$ and $21.5 \mathrm{~cd} / \mathrm{A}$ for the device $\mathrm{B}$. The luminances under an applied voltage of $10 \mathrm{~V}$ were $130 \mathrm{~cd} / \mathrm{m}^{2}$ for the device A and $950 \mathrm{~cd} / \mathrm{m}^{2}$ for the device B. The remarkable improvement of electroluminescent characteristics in the device B compared with device A comes from the better treatment of HTL in the device B than in the device A. As a HTL in the blue PhOLEDs, the TAPC could be more ideal than the conventional $\mathrm{NPB} / \mathrm{mCP}$ due to the better hole mobility, larger triplet energy and better electron confinement by a large LUMO offset at the interface of the TAPC and the emissive layer.

Figure 5 shows the current efficiency-voltage-luminance characteristic of the devices $(\mathrm{C}, \mathrm{D}, \mathrm{E})$ compared with those of device $\mathrm{B}$. The organic layer compositions of the devices (B, C, D, E) are the DNTPD/TAPC/mCP : 10\%FCNIr(pic)/ETLs. The only differences of the deivces (B, C, D, E) are the thicknesses of 3TPYMB in the $500 \AA$-thick ETL of $3 \mathrm{TPYMB} / \mathrm{Bphen}$. Among the fabricated devices, the best electroluminescent characteristics was obtained in the device B with an ETL of $3 \operatorname{TPYMB}(100 \AA) / \operatorname{Bphen}(400 \AA)$. As it can be expected, the device $\mathrm{C}$ with the Bphen only as an ETL 


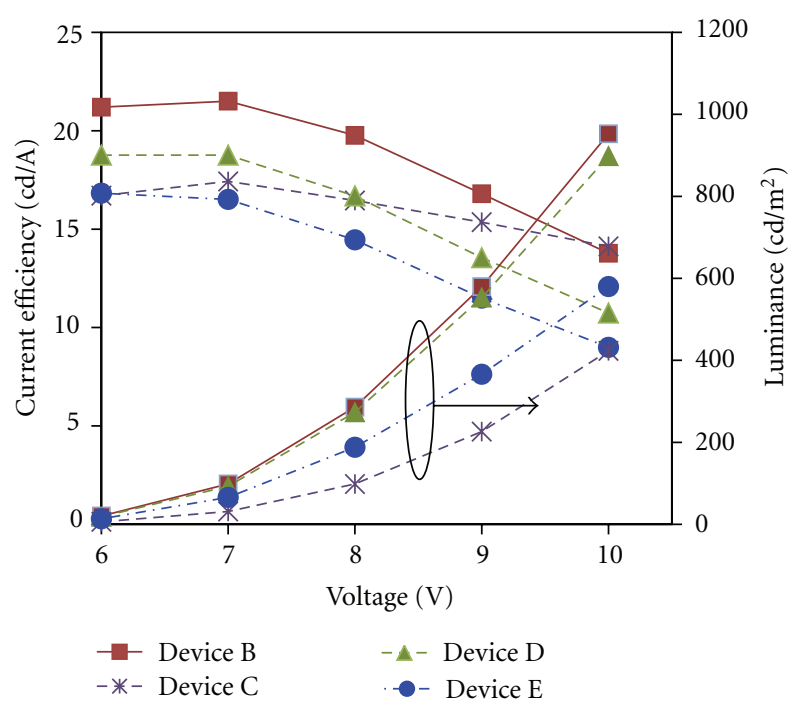

FIGURE 5: Current efficiency-voltage-luminance characteristics of the devices (C, D, E) compared with those of device B.

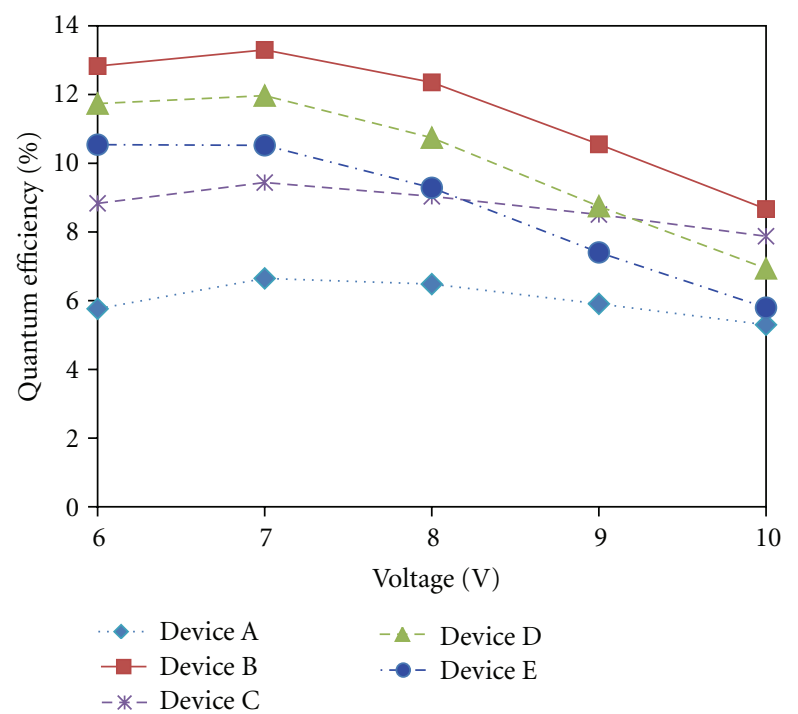

FIGURE 6: Quantum efficiency-voltage characteristics of the fabricated devices.

showed the rather poor electroluminescent characteristics because the triplet energy of Bphen is not high enough to confine the excitions. The maximum current efficiency and luminance at $10 \mathrm{~V}$ were $17,4 \mathrm{~cd} / \mathrm{A}$ and $423 \mathrm{~cd} / \mathrm{m}^{2}$ in the device $\mathrm{C}$, respectively. In the devices $(\mathrm{D}, \mathrm{E})$ with the $3 \mathrm{TPYMB}$ thicker than $100 \AA$ in the ETL, the current efficiency and luminance decreased according to the increase of 3TPYMB thickness. The maximum current efficiencies were $18.8 \mathrm{~cd} / \mathrm{A}$ for the device $\mathrm{D}$ and $16.8 \mathrm{~cd} / \mathrm{A}$ for the device $\mathrm{E}$. The luminance at $10 \mathrm{~V}$ were $900 \mathrm{~cd} / \mathrm{m}^{2}$ for the device D and $580 \mathrm{~cd} / \mathrm{m}^{2}$ for the device E. It is believed that the use of a too thick 3 TPYMB in the $3 \mathrm{TPYMB} / \mathrm{Bphen}$ results in the poor electroluminescent characteristics due to its low electron mobility.

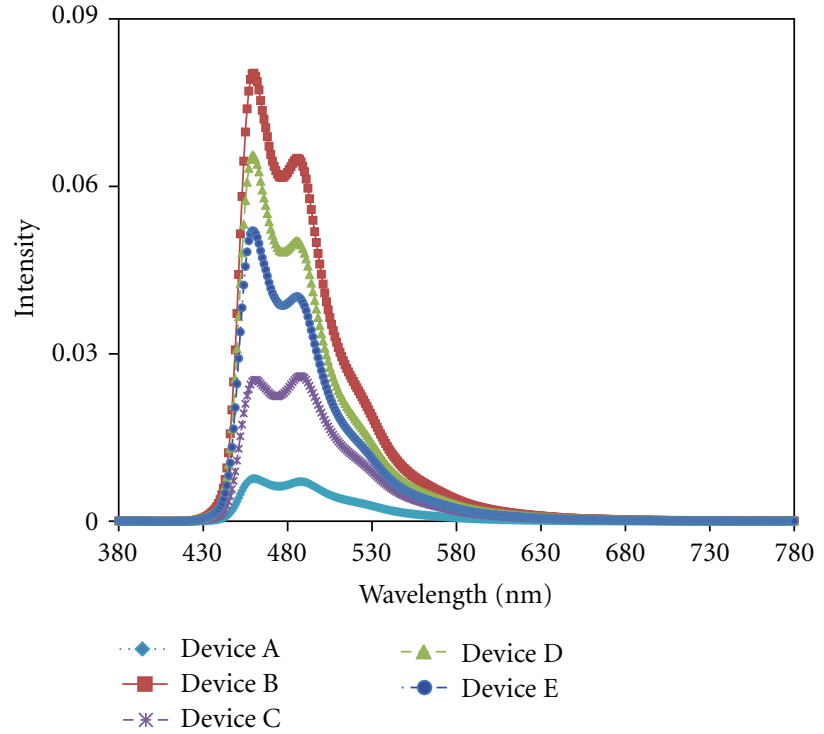

FIGURE 7: Electroluminescence spectra of the fabricated devices at $7 \mathrm{~V}$.

Figure 6 shows the quantum efficiency-voltage characteristics of the fabricated devices. The best quantum efficiencyvoltage characteristics was also obtained in the device B with a maximum quantum efficiency of $13.3 \%$ as shown in Figure 6.

The electroluminescence spectra at $7 \mathrm{~V}$ for the fabricated devices were shown in Figure 7. The Peak wavelengths were $458 \mathrm{~nm}$ and $483 \mathrm{~nm}$, which corresponds to the typical double peak emission of FCNIr(pic). The color of the fabricated devices with a central peak at $458 \mathrm{~nm}$ could be evaluated to be deep blue with the color coordinates on the CIE chart of $(0.14,0.22)$.

From the above discussion, the new proposed device of DNTPD/TAPC/mCP : 10 vol.\%FCNIr(pic)/3TPYMB/Bphen in this study can be a good blue PhOLED with high current efficiency and deep blue emission if the thicknesses of each layers are well controlled.

\section{Conclusions}

New high efficiency blue light-emitting phosphorescent devices with structure of DNPTD/HTL/mCP:FCNIr(pic)/ ETL were fabricated and evaluated according to the different treatments of HTL and ETL. A single layer of TAPC and a double layer of $\mathrm{NPB} / \mathrm{mCP}$ were compared as hole transport layers and the $3 \mathrm{TPYMB} / \mathrm{Bphen}$ layers with various thickness combinations were used as electron transport layer.

Among the fabricated devices, the device $B$ with a HTL of TAPC and an ETL of 3TPYMB $(100 \AA) / B p h e n(400 \AA)$ showed the best electroluminescent characteristics. It had a maximum current efficiency of $21.5 \mathrm{~cd} / \mathrm{A}$, and a luminance of $950 \mathrm{~cd} / \mathrm{m}^{2}$ at $10 \mathrm{~V}$.

The color coordinates were $(0.14,0.22)$ on the CIE chart, and electroluminescent spectra showed the double peak emission at $458 \mathrm{~nm}$ and $483 \mathrm{~nm}$. 
As a deep blue PhOLED with the emissive layer of mCP : FCNIr(pic), the excellent electroluminescent characteristics of device B may be obtained from the adoption of TAPC with high triplet energy as a HTL and the use of a proper thick 3TPYMB combined with Bphen as an ETL.

\section{References}

[1] B. W. D. D'Andrade and S. R. Forrest, "White organic lightemitting devices for solid-state lighting," Advanced Materials, vol. 16, no. 18, pp. 1585-1595, 2004.

[2] D. Yao, S. Zhao, J. Guo et al., "Hydroxyphenyl-benzothiazole based full color organic emitting materials generated by facile molecular modification," Journal of Materials Chemistry, vol. 21, no. 11, pp. 3568-3570, 2011.

[3] S. H. Kim, J. S. Jang, and J. Y. Lee, "High efficiency phosphorescent organic light-emitting diodes using carbazole-type triplet exciton blocking layer," Applied Physics Letters, vol. 90, no. 22, Article ID 223505, 3 pages, 2007.

[4] Z. Wu, Y. Xiong, J. L. Zou et al., "High-Triplet-Energy Poly(9,90-bis(2-ethylhexyl)-3,6-fluorene) as Host for Blue and Green Phosphorescent Complexes," Advanced Materials, vol. 20, article 2359, 2008.

[5] Y. Kawamura, K. Goushi, J. Brooks, J. Brown, H. Sasabe, and C. Adachi, "100\% phosphorescence quantum efficiency of Ir(III) complexes in organic semiconductor films," Applied Physics Letters, vol. 86, no. 7, Article ID 071104, 3 pages, 2005.

[6] Z. H. Zhao, J. Li, P. Lu, and Y. Yang, "Fluorescent, carriertrapping dopants for highly efficient single-layer polyfluorene LEDs," Advanced Functional Materials, vol. 17, no. 13, pp. 2203-2210, 2007.

[7] D. Beljonne, J. Cornil, R. H. Friend, R. A. J. Janssen, and J. L. Bredas, "Influence of chain length and derivatization on the lowest singlet and triplet states and intersystem crossing in oligothiophenes," Journal of the American Chemical Society, vol. 118, no. 27, pp. 6453-6461, 1996.

[8] K. S. Yook, S. O. Jeon, C. W. Joo, and J. Y. Lee, "High efficiency deep blue phosphorescent organic light-emitting diodes," Organic Electronics, vol. 10, no. 1, pp. 170-173, 2009.

[9] Y. M. Jeon, J. W. Kim, C. W. Lee, and M. S. Gong, "Blue organic light-emitting diodes using novel spiro[fluorenebenzofluorene]-type host materials," Dyes and Pigments, vol. 83, no. 1, pp. 66-71, 2009.

[10] Y. Zhu, A. P. Kulkarni, and S. A. Jenekhe, "Phenoxazine-Based Emissive Donor-Acceptor Materials for Efficient Organic Light-Emitting Diodes," Chemistry of Materials, vol. 17, article 5227, 2005.

[11] J. W. Lee, N. Chopra, S. H. Eom et al., "Effects of triplet energies and transporting properties of carrier transporting materials on blue phosphorescent organic light emitting devices," Applied Physics Letters, vol. 93, no. 12, Article ID 123306, 3 pages, 2008.

[12] J. S. Park, W. S. Jeon, J. H. Yu, R. Pode, and J. H. Kwon, "Efficiency optimization of green phosphorescent organic light-emitting device," Thin Solid Films, vol. 519, no. 10, pp. 3259-3263, 2011.

[13] C. Han, G. Xie, H. Xu et al., "Towards Highly Efficient Blue-Phosphorescent Organic Light-Emitting Diodes with Low Operating Voltage and Excellent Efficiency Stability," Chemistry-A EuropeanJournal, vol. 17, article 445, 2011.

[14] S. H. Eom, Y. Zheng, E. Wrzesniewski et al., "Effect of electron injection and transport materials on efficiency of deep- blue phosphorescent organic light-emitting devices," Organic Electronics, vol. 10, no. 4, pp. 686-691, 2009.

[15] S. O. Jeon, K. S. Yook, C. W. Joo, and J. Y. Lee, "Highefficiency deep-blue-phosphorescent organic light-emitting diodes using a phosphine oxide and a phosphine sulfide hightriplet-energy host material with bipolar charge-transport properties," Advanced Materials, vol. 22, no. 16, pp. 18721876, 2010. 

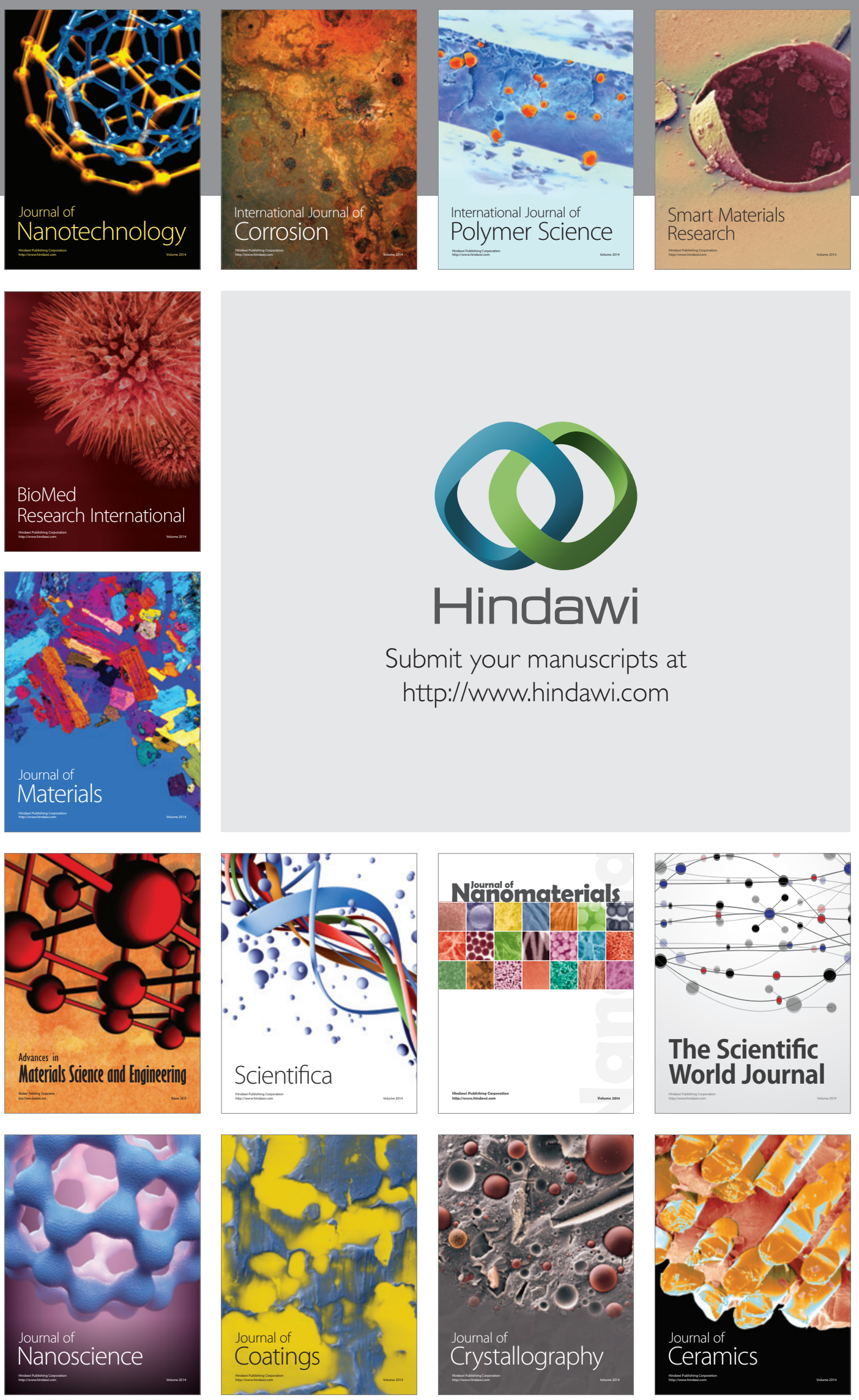

The Scientific World Journal

Submit your manuscripts at

http://www.hindawi.com

\section{World Journal}

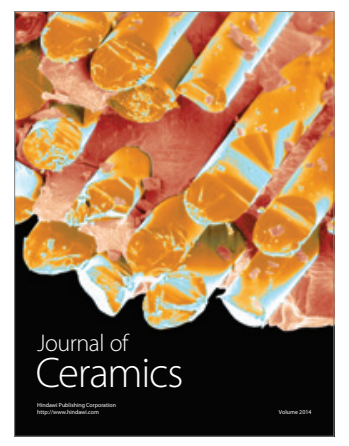

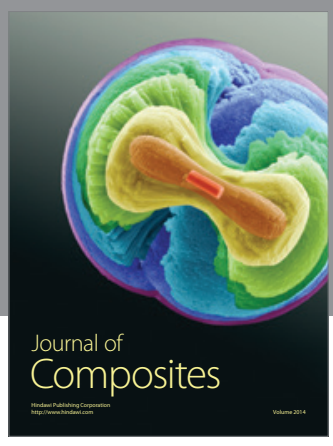
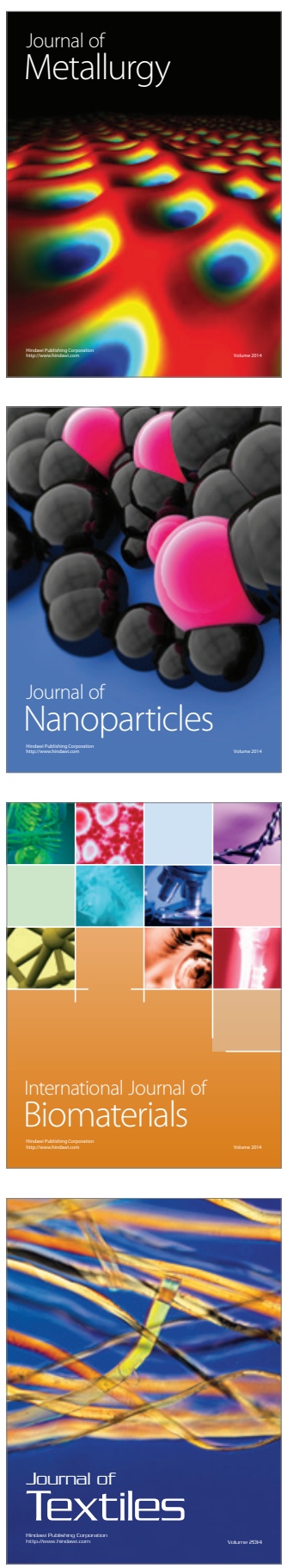